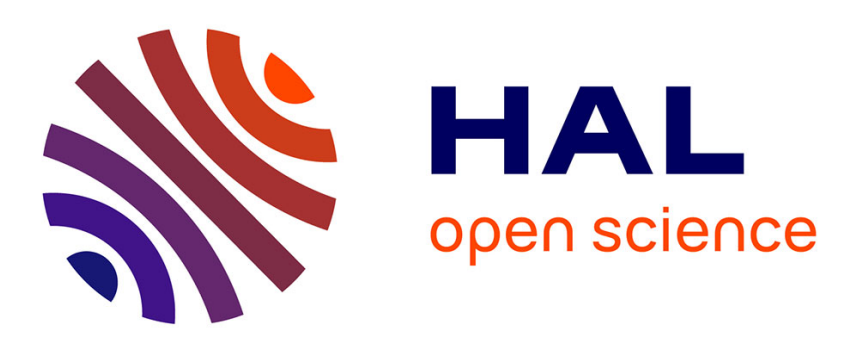

\title{
A systematic bias in fitting the surface-density profiles of interstellar filaments
}

\author{
A. P. Whitworth, F. D. Priestley, D. Arzoumanian
}

\section{To cite this version:}

A. P. Whitworth, F. D. Priestley, D. Arzoumanian. A systematic bias in fitting the surface-density profiles of interstellar filaments. Monthly Notices of the Royal Astronomical Society, 2021, 508, pp.2736-2742. 10.1093/mnras/stab2782 . hal-03585954

\section{HAL Id: hal-03585954 \\ https://hal.science/hal-03585954}

Submitted on 24 Feb 2022

HAL is a multi-disciplinary open access archive for the deposit and dissemination of scientific research documents, whether they are published or not. The documents may come from teaching and research institutions in France or abroad, or from public or private research centers.
L'archive ouverte pluridisciplinaire HAL, est destinée au dépôt et à la diffusion de documents scientifiques de niveau recherche, publiés ou non, émanant des établissements d'enseignement et de recherche français ou étrangers, des laboratoires publics ou privés. 


\title{
A systematic bias in fitting the surface-density profiles of interstellar filaments
}

\author{
A. P. Whitworth, ${ }^{1 \star}$ F. D. Priestley ${ }^{1}$ and D. Arzoumanian ${ }^{2}$ \\ ${ }^{1}$ School of Physics and Astronomy, Cardiff University, Cardiff CF24 3AA, UK \\ ${ }^{2}$ Aix Marseille Université, CNRS, CNES, LAM, Marseille, France
}

Accepted 2021 September 21. Received 2021 September 21; in original form 2021 June 30

\begin{abstract}
The surface-density profiles (SDPs) of dense filaments, in particular those traced by dust emission, appear to be well fit with Plummer profiles, i.e. $\Sigma(b)=\Sigma_{\mathrm{B}}+\Sigma_{\mathrm{O}}\left\{1+\left[b / w_{\mathrm{O}}\right]^{2}\right\}^{[1-p] / 2}$. Here, $\Sigma_{\mathrm{B}}$ is the background surface density; $\Sigma_{\mathrm{B}}+\Sigma_{\mathrm{O}}$ is the surface density on the filament spine; $b$ is the impact parameter of the line-of-sight relative to the filament spine; $w_{\mathrm{O}}$ is the Plummer scale-length (which for fixed $p$ is exactly proportional to the full width at half-maximum, $w_{\mathrm{O}}=\mathrm{FWHM} / 2\left\{2^{2 /[p-1]}-1\right\}^{1 / 2}$ ); and $p$ is the Plummer exponent (which reflects the slope of the SDP away from the spine). In order to improve signal to noise, it is standard practice to average the observed surface densities along a section of the filament, or even along its whole length, before fitting the profile. We show that, if filaments do indeed have intrinsic Plummer profiles with exponent $p_{\text {INTRINSIC }}$, but there is a range of $w_{\mathrm{O}}$ values along the length of the filament (and secondarily a range of $\Sigma_{\mathrm{B}}$ values), the value of the Plummer exponent, $p_{\text {FIT }}$, estimated by fitting the averaged profile, may be significantly less than $p_{\text {INTRINSIC }}$. The decrease, $\Delta p=p_{\text {INTRINSIC }}-p_{\text {FIT }}$, increases monotonically (i) with increasing $p_{\text {INTRINSIC }}$; (ii) with increasing range of $w_{\mathrm{O}}$ values; and (iii) if (but only if) there is a finite range of $w_{\mathrm{O}}$ values, with increasing range of $\Sigma_{\mathrm{B}}$ values. For typical filament parameters, the decrease is insignificant if $p_{\text {INTRINSIC }}=2(0.05 \lesssim \Delta p \lesssim 0.10)$, but for $p_{\text {INTRINSIC }}=3$, it is larger $(0.18 \lesssim \Delta p \lesssim 0.50)$, and for $p_{\text {INTRINSIC }}=4$, it is substantial $(0.50 \lesssim \Delta p \lesssim 1.15)$. On its own, this effect is probably insufficient to support a value of $p_{\text {INTRINSIC }}$ much greater than $p_{\text {FIT }} \simeq 2$, but it could be important in combination with other effects.
\end{abstract}

Key words: stars: formation-ISM: clouds.

\section{INTRODUCTION}

In the last decade, it has become clear that filaments play a critical role in assembling the material to form stars (e.g. Schneider \& Elmegreen 1979; Bally et al. 1987; Abergel et al. 1994; Cambrésy 1999; Myers 2009; Hacar \& Tafalla 2011; Peretto et al. 2012; Hacar et al. 2013; Palmeirim et al. 2013; Peretto et al. 2013; Alves de Oliveira et al. 2014; André et al. 2014; Könyves et al. 2015; Marsh et al. 2016; Hacar, Tafalla \& Alves 2017; Ward-Thompson et al. 2017; Hacar et al. 2018; Williams et al. 2018; Watkins et al. 2019; Ladjelate et al. 2020; Arzoumanian et al. 2021). Even in clouds that are not apparently forming stars, or forming them very slowly (e.g. Joncas, Boulanger \& Dewdney 1992; Falgarone, Pety \& Phillips 2001; McClure-Griffiths et al. 2006; Ward-Thompson et al. 2010), including The Brick in the Central Molecular Zone of the Galaxy (Federrath et al. 2016), the internal structure is still dominated by filaments.

Filaments are particularly pronounced in maps of thermal dustemission, such as those made using the Herschel Space Telescope (e.g. André et al. 2010; Men'shchikov et al. 2010; Molinari et al. 2010; Hill et al. 2011; Hennemann et al. 2012; Schneider et al. 2012; Schisano et al. 2014; Benedettini et al. 2015; Wang et al. 2015; Cox et al. 2016). Given maps of thermal dust emission at a range

^E-mail: anthony.whitworth@ astro.cf.ac.uk of different wavelengths, it is possible to derive maps of the dust optical depth, $\tau_{\mathrm{F}}$ at a fiducial far-infrared wavelength, $\lambda_{\mathrm{F}}$, either by Modified Blackbody fitting (e.g. Hill et al. 2011; Peretto et al. 2012; Schneider et al. 2012; Palmeirim et al. 2013; Alves de Oliveira et al. 2014; Benedettini et al. 2015; Wang et al. 2015; Cox et al. 2016; Ladjelate et al. 2020), or by more sophisticated techniques (e.g. Howard et al. 2019, 2021) like PPMAP (Marsh, Whitworth \& Lomax 2015; Whitworth et al. 2019). Such maps are more accurate if the range of wavelengths (a) is large, and (b) extends well above and well below the peak of the spectral energy distribution. It is also necessary that the emission at all the wavelengths used be optically thin.

If the mass opacity, $\kappa_{\mathrm{F}}$, of dust at $\lambda_{\mathrm{F}}$ is known (and universal), one can convert a map of $\tau_{\mathrm{F}}$ into a map of the surface density of dust, $\Sigma_{\mathrm{D}}=\tau_{\mathrm{F}} / \kappa_{\mathrm{F}}$. If the fraction of dust by mass, $Z_{\mathrm{D}}$ is known (and universal), one can convert this map into a map of the total surface density (hereafter simply 'the surface density'), $\Sigma=\Sigma_{\mathrm{D}} / Z_{\mathrm{D}}$. Finally, if one assumes that all the hydrogen is molecular, one can convert the map of $\Sigma$ into a map of the column-density of molecular hydrogen, $N_{\mathrm{H}_{2}}=X \Sigma / 2 m_{\mathrm{H}}$. Here, $X$ is the fraction of hydrogen by mass and $m_{\mathrm{H}}$ is the mass of an hydrogen atom. With $X=0.70$ this reduces to

$N_{\mathrm{H}_{2}}=4.4 \times 10^{19} \mathrm{~cm}^{-2}\left[\frac{\Sigma}{\mathrm{M}_{\odot} \mathrm{pc}^{-2}}\right]$.

However, this last conversion neglects the fact that on most lines of sight a significant fraction of the hydrogen is not molecular. 
Therefore, in the sequel, we prefer to present our analysis in terms of $\Sigma$.

\subsection{Plummer profiles}

Dust-emission filaments are found to have surface-density profiles (hereafter SDPs) that can be fit with Plummer profiles,

$\Sigma(b)=\Sigma_{\mathrm{B}}+\Sigma_{\mathrm{O}}\left\{1+\left[\frac{b}{w_{\mathrm{O}}}\right]^{2}\right\}^{-[p-1] / 2}$,

(e.g. Arzoumanian et al. 2011; Palmeirim et al. 2013; Cox et al. 2016; André et al. 2016; Arzoumanian et al. 2019; Howard et al. $2019,2021)$. In equation (2), $\Sigma_{\mathrm{B}}$ is the background surface density, $\Sigma_{\mathrm{B}}+\Sigma_{\mathrm{O}}$ is the surface density on the filament spine, $b$ is the impact parameter of the line-of-sight relative to the filament spine (i.e. projected distance from the filament spine), $w_{\mathrm{O}}$ is the Plummer scale-length relating to the width of the densest part of the filament, and $p$ is the Plummer exponent relating to the density gradient in the outer parts of the filament.

The full-widths at half-maximum surface density is related to $w_{\mathrm{O}}$ by

$\mathrm{FWHM} \simeq 2 w_{\mathrm{O}}\left\{2^{2 /[p-1]}-1\right\}^{1 / 2}$,

so for fixed $p$, the FWHM is exactly proportional to $w_{\mathrm{O}}$. Implicitly the Plummer exponent is

$p=1-\operatorname{LIM}_{b \rightarrow \infty}\left\{\frac{d \ln \left(\Sigma-\Sigma_{\mathrm{B}}\right)}{d \ln (b)}\right\}$.

Provided that (a) the contribution from the background $\left(\Sigma_{\mathrm{B}}\right)$ is uniform, and (b) the filament is cylindrically symmetric, the filament's underlying volume-density profile (VDP) should also subscribe to a Plummer profile, viz.

$\rho(w)=\rho_{\mathrm{B}}+\rho_{\mathrm{O}}\left\{1+\left[\frac{w}{w_{\mathrm{O}}}\right]^{2}\right\}^{-p / 2}$,

as shown by Casali (1986). Here, $\rho_{\mathrm{B}}$ is the background volumedensity, $\rho_{\mathrm{O}}$ is the excess volume-density on the filament spine, $w$ is the true (i.e. 3D) radial distance from the filament spine, and $w_{\mathrm{O}}$ is the same Plummer scale-length as invoked in equations (2) and (3). Implicitly the Plummer exponent is

$p=-\operatorname{LIM}_{w \rightarrow \infty}\left\{\frac{d \ln \left(\rho-\rho_{\mathrm{B}}\right)}{d \ln (w)}\right\}$,

and

$\rho_{\mathrm{O}}=\frac{\Sigma_{\mathrm{O}} \Gamma(p / 2) \cos (\psi)}{w_{\mathrm{O}} \Gamma(1 / 2) \Gamma(p / 2-1 / 2)} ;$

$\Gamma$ is the gamma function and $\psi$ is the angle between the filament spine and the plane of the sky.

\subsection{Longitudinally averaged filament profiles}

When fitting SDPs with Plummer profiles (i.e. equation 2), it is a standard practice to first derive a single profile averaged along the length of the whole filament or a section thereof (in order to improve signal to noise), and then to estimate the Plummer parameters that best fit these longitudinally averaged filament profiles (e.g. Arzoumanian et al. 2011, 2019; Howard et al. 2019, 2021). We distinguish parameters derived in this way with a subscript 'FIT'.

$\Sigma_{\mathrm{B} \text { : FIT }}$ and $\Sigma_{\mathrm{O} \text { : FIT }}$ can be determined directly (modulo some straightforward interpolation). There are then only two further
Table 1. The box-car distribution parameters (hereafter simply 'the distribution parameters'). The left hand column gives the fixed values of $\mu_{X}$, which represents the mean of $\log _{10}(X)$. The right hand column gives the range of $\sigma_{X}$ that we explore, where $\sigma_{X}$ represents the standard deviation of $\log _{10}(X)$. $X$ stands for $\left[\Sigma_{\mathrm{B}} / \mathrm{M}_{\odot} \mathrm{pc}^{-2}\right],\left[\Sigma_{\mathrm{O}} / \mathrm{M}_{\odot} \mathrm{pc}^{-2}\right]$ and $\left[w_{\mathrm{O}} / \mathrm{pc}\right]$.

\begin{tabular}{|c|c|}
\hline FIXED MEANS & RANGES OF STANDARD DEVIATIONS \\
\hline \multicolumn{2}{|c|}{ Background surface density, $\left[\Sigma_{\mathrm{B}} / \mathrm{M}_{\odot} \mathrm{pc}^{-2}\right]$ : } \\
\hline$\mu_{\Sigma_{\mathrm{B}}}=1.778$ & $0 \leq \sigma_{\Sigma_{\mathrm{B}}} \leq 0.40$ \\
\hline \multicolumn{2}{|c|}{ Excess surface density on filament spine $\left[\Sigma_{\mathrm{O}} / \mathrm{M}_{\odot} \mathrm{pc}^{-2}\right]$. } \\
\hline$\mu_{\Sigma_{\mathrm{O}}}=1.778$ & $0 \leq \sigma_{\Sigma_{0}} \leq 0.40$ \\
\hline \multicolumn{2}{|c|}{ Plummer scale-length of filament, $\left[w_{\mathrm{O}} / \mathrm{pc}\right]$. } \\
\hline$\mu_{w_{\mathrm{O}}}=-1.523$ & $0 \leq \sigma_{w_{\mathrm{O}}} \leq 0.40$ \\
\hline
\end{tabular}

parameters to estimate: $w_{\mathrm{O}}$ FIT (or strictly speaking its angular equivalent, $\theta_{\mathrm{O} \text { : FIT }}=w_{\mathrm{O} \text { : FIT }} / D$, where $D$ is the distance to the source); and $p_{\text {FIT }}$. Values of $w_{\text {O:FIT }} \sim 0.03 \mathrm{pc}$ (corresponding to FWHM $\sim 0.1 \mathrm{pc}$ ) and $p_{\text {FIT }} \sim 2$ are commonly reported for the filaments observed in local molecular clouds (e.g. Arzoumanian et al. 2011; Palmeirim et al. 2013; André et al. 2014; Arzoumanian et al. 2019), and also for the filaments identified in hydrodynamic and magnetohydrodynamic simulations of turbulent molecular clouds (e.g. Smith, Glover \& Klessen 2014; Kirk et al. 2015; Federrath 2016; Priestley \& Whitworth 2020).

However, Panopoulou et al. (2017) have pointed out that the distribution of intrinsic FWHM values for interstellar filaments, although centred on $\sim 0.1 \mathrm{pc}$, may be significantly broader than reported, due to the averaging process.

Here, we show that the intrinsic $p$ values of interstellar filaments (hereafter $p_{\text {INTRINSIC }}$ ) may also be larger than reported, i.e. $p_{\text {INTRINSIC }}$ $>p_{\text {FIT }}$, again due to the averaging process. Specifically, the reduction, $\Delta p=p_{\text {INTRINSIC }}-p_{\text {FIT }}$ is larger for larger values of $p_{\text {INTRINSIC. }} \Delta p$ is also larger if the range of $w_{\mathrm{O}}$ values is larger. And finally, provided there is a finite range of $w_{\mathrm{O}}$ values, $\Delta p$ is larger if the range of $\Sigma_{\mathrm{B}}$ values is larger.

We define our computational methodology in Section 2. We present our results in Section 3. We summarise our conclusions in Section 4.

\section{COMPUTATIONAL METHODOLOGY}

\subsection{Logarithmic box-car distributions}

In the sequel, SDPs are generated with the Plummer exponent held

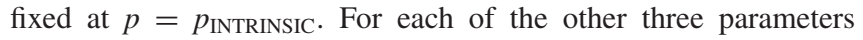
defining the Plummer SDP (i.e. $X \equiv\left[\Sigma_{\mathrm{B}} / \mathrm{M}_{\odot} \mathrm{pc}^{-2}\right],\left[\Sigma_{\mathrm{O}} / \mathrm{M}_{\odot} \mathrm{pc}^{-2}\right]$, $\left[w_{\mathrm{O}} / \mathrm{pc}\right]$; see equations 2 and 3 ) we assume that $\log _{10}(X)$ has a boxcar distribution, with mean $\mu_{X}$ and standard deviation $\sigma_{X},{ }^{1}$ i.e.

$\frac{\mathrm{d} P}{\mathrm{~d} \log _{10}(X)}= \begin{cases}\frac{\mathrm{d} \log _{10}(X)}{2 \sqrt{ } 3 \sigma_{X}}, & \left|\log _{10}(X)-\mu_{X}\right| \leq \sqrt{ } 3 \sigma_{X} \\ 0, & \left|\log _{10}(X)-\mu_{X}\right|>\sqrt{ } 3 \sigma_{X} .\end{cases}$

\subsection{Filament configurations}

For the purpose of this study, all the means $\left(\mu_{\Sigma_{\mathrm{B}}}, \mu_{\Sigma_{\mathrm{O}}}, \mu_{w_{\mathrm{O}}}\right)$ have fixed values, as given in the left hand column of Table 1. These correspond to a median background surface density, $\Sigma_{\mathrm{B}}=$ $60 \mathrm{M}_{\odot} \mathrm{pc}^{-2}$ (equivalently $N_{\mathrm{H}_{2}} \simeq 2.6 \times 10^{21} \mathrm{~cm}^{-2}$ ), a median spinal

\footnotetext{
${ }^{1}$ Strictly speaking, ' $\mu_{X}$ ' should read ' $\mu_{\log _{10}(X)}$ ', and ' $\sigma_{X}$ ' should read ' $\sigma_{\log _{10}(X)}$ '. We use the shorter version for convenience.
} 
surface density $\Sigma_{\mathrm{O}}=60 \mathrm{M}_{\odot} \mathrm{pc}^{-2}$, and a median Plummer scalelength $w_{\mathrm{O}}=0.03 \mathrm{pc}$. These choices of $\mu_{\Sigma_{\mathrm{B}}}, \mu_{\Sigma_{\mathrm{O}}}$, and $\mu_{w_{\mathrm{O}}}$ are informed by the results of Arzoumanian et al. (2019).

The different filament configurations that we explore, are therefore, completely defined by specifying the three standard deviations: $\sigma_{\Sigma_{\mathrm{B}}}, \sigma_{\Sigma_{\mathrm{O}}}$, and $\sigma_{w_{\mathrm{O}}}$. We consider values for these standard deviations within the limits specified in the right hand column of Table 1. A large standard deviation means that the associated parameter varies over a large range. The maximum standard deviations considered allow $\Sigma_{\mathrm{B}}$ and $\Sigma_{\mathrm{O}}$ to take values between 12 and $300 \mathrm{M}_{\odot} \mathrm{pc}^{-2}$; and $w_{\mathrm{O}}$ to take values between 0.006 and $0.15 \mathrm{pc}$. We are not suggesting that such extreme values of $\Sigma_{\mathrm{B}}, \Sigma_{\mathrm{O}}$, and $w_{\mathrm{O}}$ are the norm. Large values are treated in order to evaluate trends accurately. We discuss in Appendix A (see Table A1), the standard deviations that are actually observed.

\subsection{Multiple realisations and longitudinally averaged profiles}

For each filament configuration (i.e. each specific combination of $\sigma_{\Sigma_{\mathrm{B}}}, \sigma_{\Sigma_{\mathrm{O}}}$, and $\sigma_{w_{\mathrm{O}}}$ ), we generate $c_{\mathrm{TOT}}=10^{6}$ different random combinations of $\Sigma_{\mathrm{B}}, \Sigma_{\mathrm{O}}$, and $w_{\mathrm{O}}$. For example, different values of $\Sigma_{\mathrm{O}}$ are obtained by generating linear random deviates, $\mathcal{L}$, on the interval $[0,1]$, and then setting

$\Sigma_{\mathrm{O}}=10^{\mu \Sigma_{\mathrm{O}}+\sqrt{ } 3 \sigma_{\Sigma_{\mathrm{O}}}[2 \mathcal{L}-1]}$.

Each combination of $\Sigma_{\mathrm{B}}, \Sigma_{\mathrm{O}}$, and $w_{\mathrm{O}}$ allows us to compute an individual SDP, and these individual SDPs are added and normalised to produce a longitudinally averaged SDP, $\Sigma_{\mathrm{FIT}}(b)$, for that filament configuration. The results presented below involve $\sim 5 \times 10^{5}$ different filament configurations, and hence $\sim 5 \times 10^{11}$ individual SDPs.

\subsection{Plummer profile fitting}

The average SDP, $\bar{\Sigma}_{\mathrm{CONFIG}}(b)$, for a given filament configuration, $\left[\sigma_{\Sigma_{\mathrm{B}}}, \sigma_{\Sigma_{\mathrm{O}}}, \sigma_{w_{\mathrm{O}}}\right]$, is fit with a Plummer-profile (equation 2), and the best-fit parameters, $\left(\Sigma_{\mathrm{B}: \text { FIT }}, \Sigma_{\mathrm{O}: \text { FIT }}\right.$, and $\left.w_{\mathrm{O} \text { : FIT }}\right)$ are established to five significant figures. The quality of the fit is measured with the fractional root mean square error, $\mathcal{Q}_{\mathrm{FIT}}$, given by

$$
\begin{aligned}
\mathcal{Q}_{\mathrm{FIT}}^{2}= & \frac{1}{i_{\mathrm{TOT}}} \sum_{i=1}^{i=i_{\mathrm{TOT}}}\left\{\frac { 1 } { \overline { \Sigma } _ { \mathrm { CONFIG } } ^ { 2 } ( b _ { i } ) } \left[\bar{\Sigma}_{\mathrm{CONFIG}}\left(b_{i}\right)-\Sigma_{\mathrm{B}: \mathrm{FIT}}\right.\right. \\
& \left.\left.-\Sigma_{\mathrm{O}: \mathrm{FIT}}\left\{1+\left[\frac{b_{i}}{w_{\mathrm{O}: \mathrm{FIT}}}\right]^{2}\right\}^{-\left[p_{\mathrm{FIT}}-1\right] / 2}\right]^{2}\right\} .
\end{aligned}
$$

Here, the $b_{i}\left(i=1\right.$ to $\left.i_{\mathrm{TOT}}=401\right)$ are impact parameters uniformly spaced between $b_{1}=0.000 \mathrm{pc}$ and $b_{401}=0.400 \mathrm{pc}$.

\subsection{Correlated Plummer parameters}

Arzoumanian et al. (2019) note that the surface density on the filament spine is correlated with the background surface density (see their fig. $6 \mathrm{c}$ and the associated caption). Specifically they find

$N_{\mathrm{H}_{2}: \mathrm{O}} \simeq[0.95 \pm 0.15] N_{\mathrm{H}_{2}: \mathrm{B}}-[0.15 \pm 0.39] \times 10^{21} \mathrm{~cm}^{-3}$,

where $N_{\mathrm{H}_{2}: \mathrm{B}}+N_{\mathrm{H}_{2}: \mathrm{O}}$ is the column-density of molecular hydrogen on the filament spine, and $N_{\mathrm{H}_{2}: \mathrm{B}}$ is the column-density of molecular hydrogen in the background. We have therefore, repeated our analysis with the equation for generating values of $\Sigma_{O}$ (i.e. equation 9) replaced by the equivalent equation in terms of surface density:

$\Sigma_{\mathrm{O}}=\left\{0.95 \Sigma_{\mathrm{B}}-3.4 \mathrm{M}_{\odot} \mathrm{pc}^{-2}\right\} 10^{\sqrt{3} \sigma_{\Sigma_{\mathrm{O}}}[2 \mathcal{L}-1]}$.
In Appendix A, we discuss the Arzoumanian et al. (2019) data set in more detail, and possible reasons for this correlation. We do not consider correlations between any of the other pairs of Plummer distribution parameters.

\section{RESULTS}

We are concerned here with the values of $p_{\text {FIT }}$ for a longitudinally averaged SDP when there are significant variations in the background surface density, $\Sigma_{\mathrm{B}}$, and/or the spinal surface density, $\Sigma_{\mathrm{O}}$, and/or the radial scale-length, $w_{\mathrm{O}}$, along the filament or section of filament being considered.

We label $w_{\mathrm{O}}$ the primary parameter, because a finite range of $w_{\mathrm{O}}$ values always produces a reduction in $p_{\mathrm{FIT}}$, irrespective of whether there is variation in $\Sigma_{\mathrm{B}}$ or $\Sigma_{\mathrm{O}}$. The reduction increases with increasing range (i.e. increasing $\sigma_{w_{\mathrm{O}}}$ ).

We label $\Sigma_{\mathrm{B}}$ the secondary parameter, because a finite range of $\Sigma_{\mathrm{B}}$ values only produces a reduction in $p_{\text {FIT }}$ when there is also a finite range of $w_{\mathrm{O}}$ values. The associated reduction is relatively small, and increases with both the range of $w_{\mathrm{O}}$ values and the range of $\Sigma_{\mathrm{B}}$ values (i.e. increasing $\sigma_{w_{\mathrm{O}}}$ and increasing $\sigma_{\Sigma_{\mathrm{B}}}$ ). The reduction associated with the range of $\Sigma_{\mathrm{B}}$ values tends to saturate at large $\sigma_{\Sigma_{\mathrm{B}}}$

We label $\Sigma_{\mathrm{O}}$ the null parameter, because whatever the range of $\Sigma_{\mathrm{O}}$ values it has no effect on $p_{\text {FIT }}$.

\subsection{One parameter at a time}

To demonstrate these dependences, we first consider one parameter at a time, and increase the range of that parameter while keeping the other two parameters fixed. In other words, we vary one of $\sigma_{\Sigma_{\mathrm{B}}}, \sigma_{\Sigma_{\mathrm{O}}}$, and $\sigma_{w_{\mathrm{O}}}$ in turn, and set the other two to zero.

If we increase $\sigma_{\Sigma_{\mathrm{B}}}$ (i.e. we increase the range of $\Sigma_{\mathrm{B}}$ ), with $\sigma_{\Sigma_{\mathrm{O}}}=$ $\sigma_{w_{\mathrm{O}}}=0$ (i.e. fixed $\Sigma_{\mathrm{O}}=60 \mathrm{M}_{\odot} \mathrm{pc}^{-2}$ and fixed $w_{\mathrm{O}}=0.03 \mathrm{pc}$ ), this has no effect on $p_{\text {FIT }}$, which remains exactly equal to $p_{\text {INTRINSIC. }}$.

Likewise, if we increase $\sigma_{\Sigma_{\mathrm{O}}}$ (i.e. we increase the range of $\Sigma_{\mathrm{O}}$ ), with $\sigma_{\Sigma_{\mathrm{B}}}=\sigma_{w_{\mathrm{O}}}=0$ (i.e. fixed $\Sigma_{\mathrm{B}}=60 \mathrm{M}_{\odot} \mathrm{pc}^{-2}$ and fixed $w_{\mathrm{O}}=$ $0.03 \mathrm{pc}$ ), this too has no effect on $p_{\text {FIT }}$, which remains exactly equal to $p_{\text {INTRINSIC }}$.

However, if we increase $\sigma_{w_{\mathrm{O}}}$ (i.e. we increase the range of $w_{\mathrm{O}}$ ), with $\sigma_{\Sigma_{\mathrm{B}}}=\sigma_{\Sigma_{\mathrm{O}}}=0$ (i.e. fixed $\Sigma_{\mathrm{B}}=60 \mathrm{M}_{\odot} \mathrm{pc}^{-2}$ and fixed $\Sigma_{\mathrm{O}}=$ $\left.60 \mathrm{M}_{\odot} \mathrm{pc}^{-2}\right), p_{\text {FIT }}$ is reduced, as shown on Fig. 1. $w_{\mathrm{O}}$ is therefore, the primary parameter on the grounds that it is the only parameter whose variation, on its own, affects $p_{\text {FIT }}$. Moreover, it affects $p_{\text {FIT }}$ strongly if $p_{\text {INTRINSIC }}$ is large.

\subsection{Two parameters at a time}

Next we consider two parameters at a time, and vary the ranges of those two parameters simultaneously while keeping the value of the third parameter fixed at its default value. In other words, we vary two of $\sigma_{\Sigma_{\mathrm{B}}}, \sigma_{\Sigma_{\mathrm{O}}}$, and $\sigma_{w_{\mathrm{O}}}$, and set the remaining one to zero.

If we vary the range of $\Sigma_{\mathrm{B}}$ (i.e. vary $\sigma_{\Sigma_{\mathrm{B}}}$ ) and simultaneously vary the range of $\Sigma_{\mathrm{O}}$ (i.e. vary $\sigma_{\Sigma_{\mathrm{O}}}$ ), with $w_{\mathrm{O}}$ fixed at its default value (i.e. $w_{\mathrm{O}}=0.03 \mathrm{pc}$ ), the value of $p_{\text {FIT }}$ is unaffected, and remains at $p_{\text {INTRINSIC. }}$.

If we vary the range of $\Sigma_{\mathrm{B}}$ (i.e. vary $\sigma_{\Sigma_{\mathrm{B}}}$ ) and simultaneously vary the range of $w_{\mathrm{O}}$ (i.e. vary $\sigma_{w_{\mathrm{O}}}$ ), with $\Sigma_{\mathrm{O}}$ fixed at its default value (i.e. $\Sigma_{\mathrm{O}}=60 \mathrm{M}_{\odot} \mathrm{pc}^{-2}$ ), both variations produce a change in $p_{\text {FIT }}$ Fig. 2 shows contours of constant $p_{\text {FIT }}$ on the $\left(\sigma_{w_{\mathrm{O}}}, \sigma_{\Sigma_{\mathrm{B}}}\right)$ plane, for filaments with $p_{\text {INTRINSIC }}=4$; similarly Figs 3 and 4 show the analogous results for $p_{\text {INTRINSIC }}=3$ and $p_{\text {INTRINSIC }}=2$, respectively. In each case, we see that $w_{\mathrm{O}}$ is the primary parameter; at fixed $\sigma_{\Sigma_{\mathrm{B}}}$, 


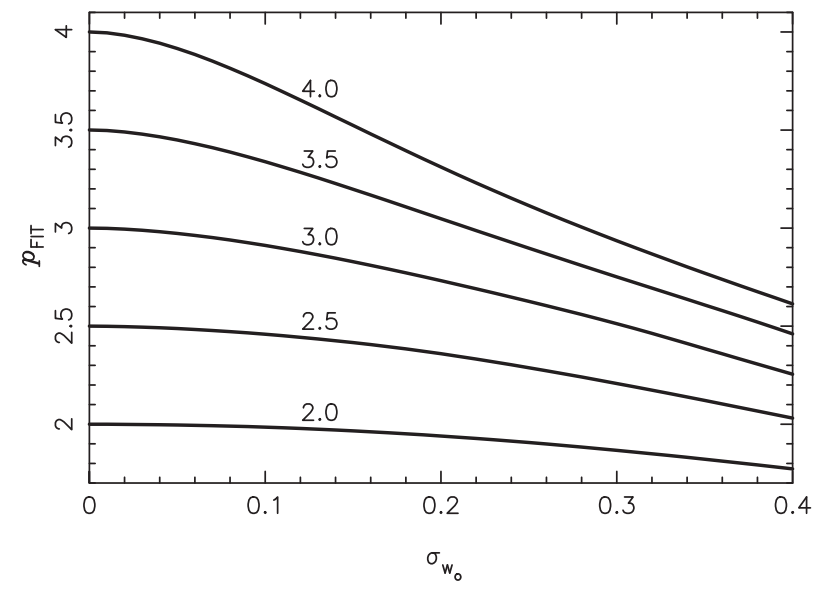

Figure 1. The variation of $p_{\text {FIT }}$ with $\sigma_{w_{\mathrm{O}}}$ when the other ranges are set to zero $\left(\sigma_{\Sigma_{\mathrm{B}}}=\sigma_{\Sigma_{\mathrm{O}}}=0\right)$. Results are shown for $p_{\text {INTRINSIC }}=$ 4.0, 3.5, 3.0, 2.5, and 2.0, as labelled. For each curve, 401 profiles have been generated and fitted. For the $p_{\text {INTRINSIC }}=4.0,3.5,3.0,2.5$, and 2.0 curves the average fractional root-mean-square error is $\overline{\mathcal{Q}}=0.012( \pm 0.011), 0.012( \pm$ $0.010), 0.010( \pm 0.008), 0.008( \pm 0.005)$, and $0.006( \pm 0.004)$, respectively (see equation 10).

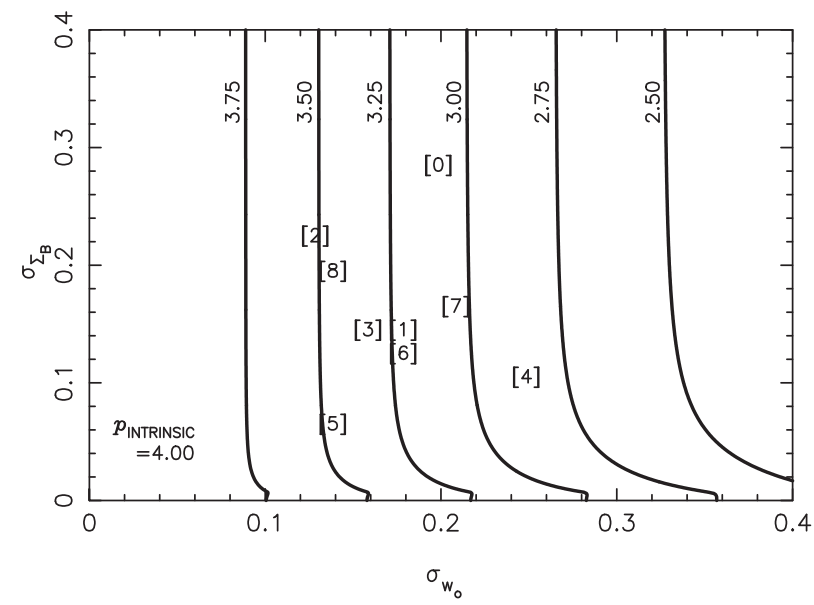

Figure 2. Contours of constant $p_{\text {FIT }}$ on the $\left[\sigma_{w_{\mathrm{O}}}, \sigma_{\Sigma_{\mathrm{B}}}\right]$ plane for $p_{\text {INTRINSIC }}=$ 4. The $1.6 \times 10^{5}$ profiles generated and fitted for this plot have an average fractional root mean square error $\overline{\mathcal{Q}}=0.0012( \pm 0.0034)$ (see equation 10). The numbers in square brackets represent the values for filaments in different regions: [0] All regions; [1] IC5146; [2] Orion B; [3] Aquila; [4] Musca; [5] Polaris; [6] Pipe; [7] Taurus L1495; [8] Ophiuchus (see Appendix A for details).

$p_{\text {FIT }}$ decreases monotonically and relatively rapidly with increasing $\sigma_{w_{\mathrm{O}}}$, especially for larger $p_{\text {INTRINSIC. }} \Sigma_{\mathrm{B}}$ is the secondary parameter: At fixed but finite $\sigma_{w_{\mathrm{O}}}, p_{\text {FIT }}$ decreases monotonically but relatively slowly with increasing $\sigma_{\Sigma_{\mathrm{B}}}$, and tends towards a constant asymptotic value.

Finally, if we vary the range of $\Sigma_{\mathrm{O}}$ (i.e. vary $\sigma_{\Sigma_{\mathrm{O}}}$ ) and simultaneously vary the range of $w_{\mathrm{O}}$ (i.e. vary $\sigma_{w_{\mathrm{O}}}$ ), with $\Sigma_{\mathrm{B}}$ fixed at it default value (i.e. $\Sigma_{\mathrm{B}}=60 \mathrm{M}_{\odot} \mathrm{pc}^{-2}$ ), we find that $p_{\text {FIT }}$ is completely independent of the value of $\sigma_{\Sigma_{0}}$, and depends on $\sigma_{w_{\mathrm{O}}}$ in exactly the same way as when $\sigma_{w_{0}}$ was varied on its own, i.e. as shown on Fig. 1. Thus, $\Sigma_{\mathrm{O}}$ is a null parameter: Under no circumstance does its range have an effect on $p_{\text {FIT }}$.

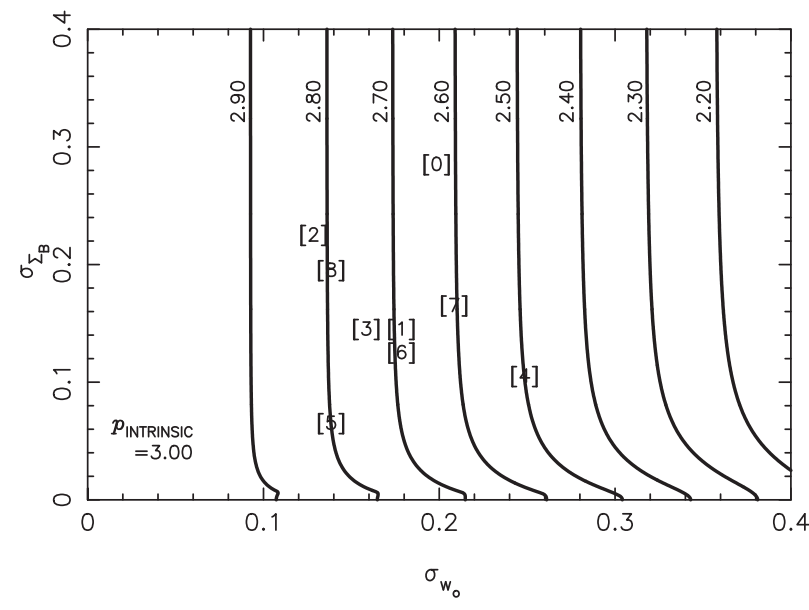

Figure 3. As Fig. 2, but for $p_{\text {INTRINSIC }}=3 ; \overline{\mathcal{Q}}=0.0011( \pm 0.0028)$.

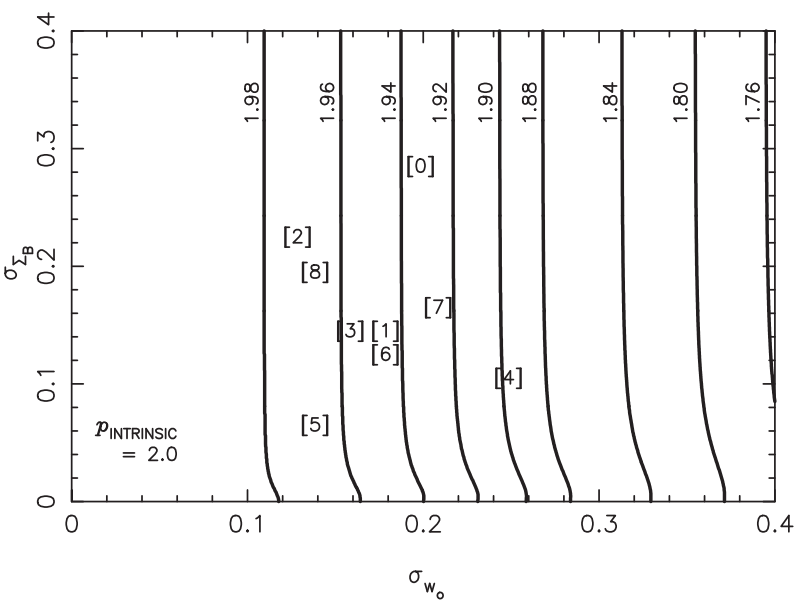

Figure 4. As Fig. 2, but for $p_{\text {INTRINSIC }}=2 ; \overline{\mathcal{Q}}=0.0006( \pm 0.0016)$.

\subsection{Effect of correlation between $\Sigma_{O}$ and $\Sigma_{B}$}

If we include the correlation between $\Sigma_{\mathrm{O}}$ and $\Sigma_{\mathrm{B}}$ (i.e. we generate values of $\Sigma_{O}$ using equation 12 rather than equation 9), the results are unchanged. This is unsurprising, since $\Sigma_{\mathrm{O}}$ is the null parameter.

\subsection{Comparison with observational data}

The numbers in square brackets on Figs 2, 3, and 4 show the values of $\sigma_{w_{\mathrm{O}}}$ and $\sigma_{\Sigma_{\mathrm{B}}}$ estimated for the different regions analysed by Arzoumanian et al. (2019), as per the key in the caption to Fig. 2 and Table A1 in Appendix A.

As treated here, the effect we have evaluated appears insufficient to reduce $p_{\text {INTRINSIC }}$ from $p_{\text {INTRINSIC }}=4.0$ (as appropriate for an isolated, infinitely ong, isothermal filament in hydrostatic equilibrium, Ostriker 1964) to $p_{\text {FIT }} \sim 2$ (as reported by e.g. Arzoumanian et al. 2011; Palmeirim et al. 2013; André et al. 2014; Federrath et al. 2016; Arzoumanian et al. 2019). However, we should note that Howard et al. (2019) find - on the basis of high-resolution maps derived using PPMAP - that they can obtain a better fit to small local sections of the L1495 filament in Taurus with $p_{\text {FIT }}=4$ rather than $p_{\text {FIT }}=2$.

A more accurate evaluation should take into account two factors. First, the averaging applied by Arzoumanian et al. (2019) only involves the profiles for individual filaments, and the range of $w_{\mathrm{O}}$ values for an individual filament is likely to be lower than the range 
for the ensemble of all the filaments in a particular region. Correcting for this will decrease $\Delta p$. Second, the averaging over the ensemble of filaments will reduce the range of $w_{\mathrm{O}}$ values, as shown by Panopoulou et al. (2017), and correcting for this will increase $\Delta p . \Delta p$ will always be positive, so $p_{\text {FIT }}$ will always be less than $p_{\text {INTRINSIC. }}$

\section{CONCLUSIONS}

We have shown that averaging filament profiles can reduce the fitted Plummer exponent, $p_{\text {FIT }}$ below its intrinsic value, $p_{\text {INTRINSIC, }}$ i.e. it artificially reduces the slope of the SDS at large distance from the spine. (It is tempting to speculate that this effect operates even if the intrinsic SDP is not well fit by a Plummer profile, but we have not proven this.)

The amount of reduction is largely determined by the intrinsic Plummer exponent, $p_{\text {INTRINSIC, }}$ and the range of Plummer scale lengths, $w_{\mathrm{O}}$, with a small additional contribution from the range of background surface densities, $\Sigma_{\mathrm{B}}$.

This reduction is not affected by the apparent correlation between the the spinal surface density, $\Sigma_{\mathrm{O}}$ and the background surface density, $\Sigma_{\mathrm{B}}$, as reported in Arzoumanian et al. (2019). In Appendix A, we explore the causes of this correlation, and suggest that it may be largely a selection effect.

For the ranges reported by Arzoumanian et al. (2019), the effect we have evaluated cannot, on its own, support values of $p_{\text {INTRINSIC }}$ significantly greater than $p_{\mathrm{FIT}}=2$. Specifically, it appears that $p_{\text {INTRINSIC }} \simeq 4.0$ is only reduced to $p_{\text {FIT }} \gtrsim 3.0$ (see numbers in square brackets on Fig. 2 ), and $p_{\text {INTRINSIC }} \simeq 3.0$ is only reduced to $p_{\text {FIT }} \gtrsim 2.5$ (numbers on Fig. 3).

However, if there were some other effect that operated in tandem with the one we have evaluated, then values of $p_{\text {INTRINSIC significantly }}$ greater than $p_{\text {FIT }} \simeq 2$ might be plausible.

\section{ACKNOWLEDGEMENTS}

APW and FDP gratefully acknowledge the support of an STFC Consolidated Grant (ST/K00926/1). DA acknowledges financial support from the CNRS. We thank the referee for a careful and constructive report on the initial version of the paper which resulted in significant refinements.

\section{DATA AVAILABILITY}

The provenance of the data used in this paper is described in Arzoumanian et al. (2019). All software used will be supplied on request to $\mathrm{APW}$.

\section{REFERENCES}

Abergel A., Boulanger F., Mizuno A., Fukui Y., 1994, ApJ, 423, L59

Alves de Oliveira C. et al., 2014, A\&A, 568, A98

André P. et al., 2010, A\&A, 518, L102

André P., Di Francesco J., Ward-Thompson D., Inutsuka S. I., Pudritz R. E., Pineda J. E., 2014, in Beuther H., Klessen R. S., Dullemond C. P., Henning T., eds, Protostars and Planets VI. University of Arizona Press, Tucson, AZ, p. 27

André P. et al., 2016, A\&A, 592, A54

Arzoumanian D. et al., 2011, A\&A, 529, L6

Arzoumanian D. et al., 2019, A\&A, 621, A42

Arzoumanian D. et al., 2021, A\&A, 647, A78

Bally J., Langer W. D., Stark A. A., Wilson R. W., 1987, ApJ, 312, L45

Benedettini M. et al., 2015, MNRAS, 453, 2036

Cambrésy L., 1999, A\&A, 345, 965
Casali M. M., 1986, MNRAS, 223, 341

Cox N. L. J. et al., 2016, A\&A, 590, A110

Falgarone E., Pety J., Phillips T. G., 2001, ApJ, 555, 178

Federrath C., 2016, MNRAS, 457, 375

Federrath C. et al., 2016, ApJ, 832, 143

Hacar A., Tafalla M., 2011, A\&A, 533, A34

Hacar A., Tafalla M., Kauffmann J., Kovács A., 2013, A\&A, 554, A55

Hacar A., Tafalla M., Alves J., 2017, A\&A, 606, A123

Hacar A., Tafalla M., Forbrich J., Alves J., Meingast S., Grossschedl J., Teixeira P. S., 2018, A\&A, 610, A77

Hennemann M. et al., 2012, A\&A, 543, L3

Hill T. et al., 2011, A\&A, 533, A94

Howard A. D. P., Whitworth A. P., Marsh K. A., Clarke S. D., Griffin M. J., Smith M. W. L., Lomax O. D., 2019, MNRAS, 489, 962

Howard A. D. P., Whitworth A. P., Griffin M. J., Marsh K. A., Smith M. W. L., 2021, MNRAS, in press

Joncas G., Boulanger F., Dewdney P. E., 1992, ApJ, 397, 165

Kirk H., Klassen M., Pudritz R., Pillsworth S., 2015, ApJ, 802, 75

Könyves V. et al., 2015, A\&A, 584, A91

Ladjelate B. et al., 2020, A\&A, 638, A74

McClure-Griffiths N. M., Dickey J. M., Gaensler B. M., Green A. J., Haverkorn M., 2006, ApJ, 652, 1339

Marsh K. A., Whitworth A. P., Lomax O., 2015, MNRAS, 454, 4282

Marsh K. A. et al., 2016, MNRAS, 459, 342

Men'shchikov A. et al., 2010, A\&A, 518, L103

Molinari S. et al., 2010, A\&A, 518, L100

Myers P. C., 2009, ApJ, 700, 1609

Ostriker J., 1964, ApJ, 140, 1056

Palmeirim P. et al., 2013, A\&A, 550, A38

Panopoulou G. V., Psaradaki I., Skalidis R., Tassis K., Andrews J. J., 2017, MNRAS, 466, 2529

Peretto N. et al., 2012, A\&A, 541, A63

Peretto N. et al., 2013, A\&A, 555, A112

Priestley F. D., Whitworth A. P., 2020, MNRAS, 499, 3728

Schisano E. et al., 2014, ApJ, 791, 27

Schneider S., Elmegreen B. G., 1979, ApJS, 41, 87

Schneider N. et al., 2012, A\&A, 540, L11

Smith R. J., Glover S. C. O., Klessen R. S., 2014, MNRAS, 445, 2900

Wang K., Testi L., Ginsburg A., Walmsley C. M., Molinari S., Schisano E., 2015, MNRAS, 450, 4043

Ward-Thompson D. et al., 2010, A\&A, 518, L92

Ward-Thompson D. et al., 2017, ApJ, 842, 66

Watkins E. J., Peretto N., Marsh K., Fuller G. A., 2019, A\&A, 628, A21

Whitworth A. P. et al., 2019, MNRAS, 489, 5436

Williams G. M., Peretto N., Avison A., Duarte-Cabral A., Fuller G. A., 2018, A\&A, 613, A11

\section{APPENDIX: THE ARZOUMANIAN ET AL. (2019) DATA-SET}

Table A1 gives values of the distribution parameters derived from the large sample analysed by Arzoumanian et al. (2019), viz. $\mathcal{N}$ (the number of filaments analysed); $\mu_{\mathrm{FWHM}}$; and $\sigma_{\mathrm{FWHM}}$ (the mean and standard deviation for the logarithm of the full-width at halfmaximum); $\mu_{\Sigma_{\mathrm{B}}}$ and $\sigma_{\Sigma_{\mathrm{B}}}$ (the mean and standard deviation for the background surface density); and $\mu_{\Sigma_{\mathrm{O}}}$ and $\sigma_{\Sigma_{\mathrm{O}}}$ (the mean and standard deviation for the spinal surface density). The values of $\sigma$ are taken at their face value, although we note the arguments in Panopoulou et al. (2017) suggesting that they may be underestimates. (Since for a given $p_{\text {INTRINSIC }}$, the FWHM is proportional to $w_{\mathrm{O}}$, we take the standard deviation of the logarithm of $w_{\mathrm{O}}$ to be the same as the standard deviation of the logarithm of FWHM.)

Fig. A1 shows the values of $\Sigma_{\mathrm{O}}$ and $\Sigma_{\mathrm{B}}$ for individual filaments. This is essentially the same as fig. 6c in Arzoumanian et al. (2019), except that the axes are $\Sigma$ rather than $N_{\mathrm{H}_{2}}$ (see equation 1), and they are scaled equally. We have over-plotted (a) with a solid line the 


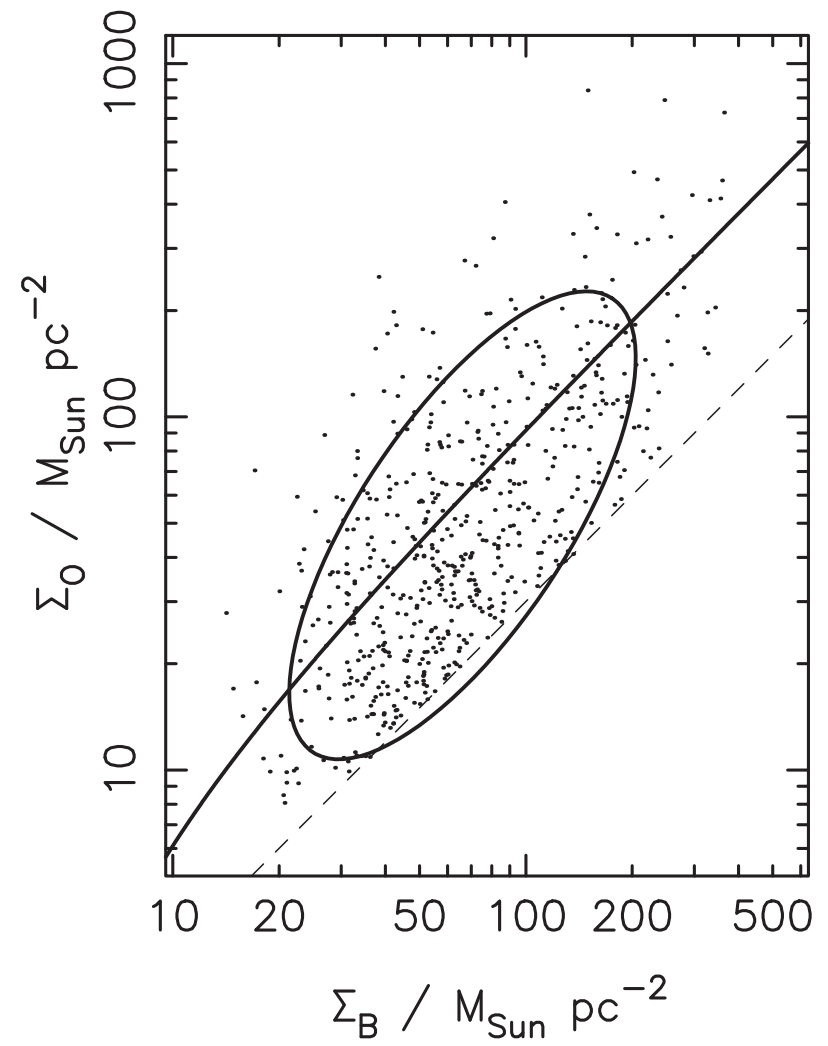

Figure A1. The distribution of profile parameters on the $\left(\sigma_{\Sigma_{\mathrm{B}}}, \sigma_{\Sigma_{\mathrm{O}}}\right)$ plane, and the corresponding moment ellipse (see the text for definition). The solid straight line shows the correlation determined by Arzoumanian et al. (2019), and the dashed line marks the contrast threshold used by Arzoumanian et al. (2019) to define a filament.

correlation derived by Arzoumanian et al. (2019),

$\Sigma_{\mathrm{O}}=0.95 \Sigma_{\mathrm{B}}-3.4 \mathrm{M}_{\odot} \mathrm{pc}^{-2}$,

(b) with a dashed straight line the contrast threshold adopted by Arzoumanian et al. (2019),

$C_{\mathrm{O}} \equiv \Sigma_{\mathrm{O}} / \Sigma_{\mathrm{B}}>0.3$,

and (c) with a solid line the 'moment ellipse'.

The moment ellipse is the ellipse which, if the same number of points were distributed uniformly within its boundary, would have the same centre of mass as the actual points, the same principal moments and the same principal axes. This is an alternative way of displaying a linear correlation between two variables (or their logarithms). It has the merit that it treats the two variables equivalently, i.e. it does not assume that one is dependent and the other independent.

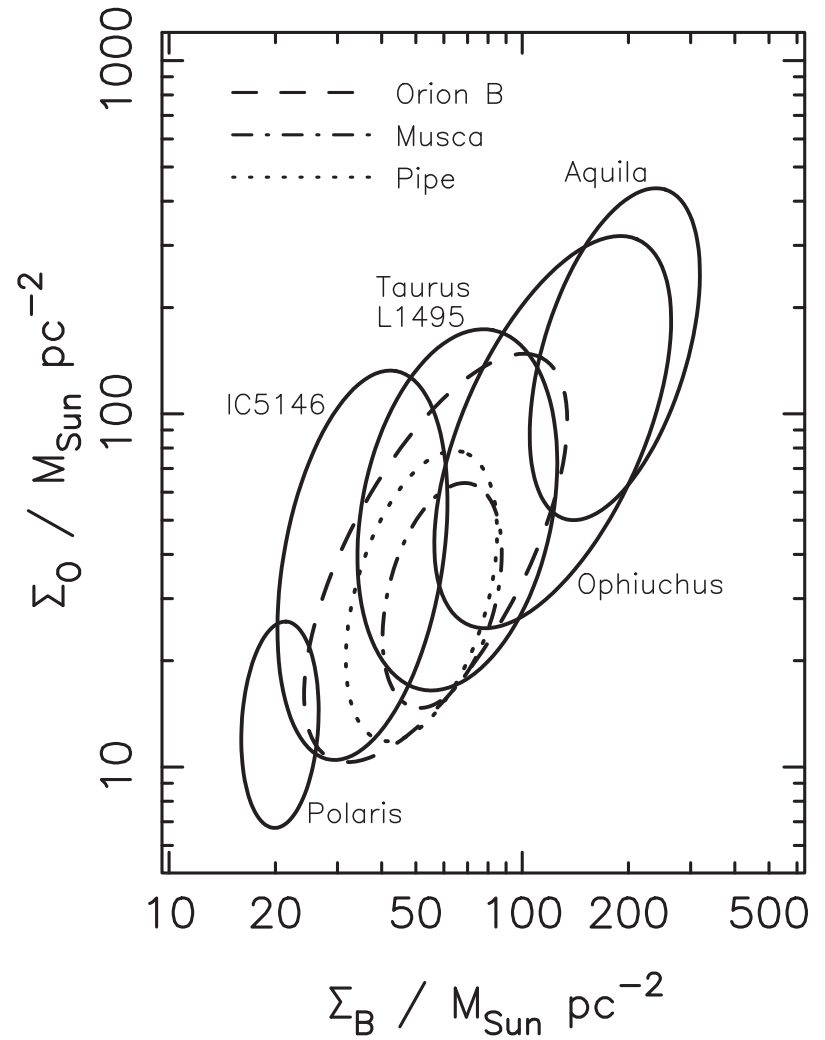

Figure A2. Moment ellipses on the $\left(\sigma_{\Sigma_{\mathrm{B}}}, \sigma_{\Sigma_{\mathrm{O}}}\right)$ plane for the different fields.

There are two key things to note about this plot. First, the columndensity contrast threshold that Arzoumanian et al. (2019) apply, accounts for the lower cut-off in values of $\Sigma_{\mathrm{O}}$, and is probably responsible for a significant part of the observed correlation. Second, the empty bit of the ellipse below this threshold is to some extent compensated by a concentration of points immediately above the threshold.

Fig. A2 shows the moment ellipses for the individual fields. It shows that within individual fields, the correlation between $\Sigma_{\mathrm{O}}$ and $\Sigma_{\mathrm{B}}$ varies. First, it is always steeper than for the ensemble of all the fields. In general, the spread of $\Sigma_{O}$ values is larger than the spread of $\Sigma_{\mathrm{B}}$ values. Second, the cases where this is less marked are those more affected by the contrast threshold. Third, the extent of the correlation noted by Arzoumanian et al. (2019) can be attributed to systematic changes from one field to another, with Polaris at one extreme, Aquila at the other extreme, and Musca and Pipe in the middle (and having very little overlap with either Polaris or Aquila). 
Table A1. Column 1 gives the name of the field considered, preceded by the number (in square brackets) used to represent this field on Figs 2, 3, and 4. Column 2 gives the number of filaments. Columns 3 and 4 give the mean, $\mu_{\mathrm{FWHM}}$, and standard deviation, $\sigma_{\mathrm{FWHM}}$, of $\log _{10}(\mathrm{FWHM} / \mathrm{pc})$, where FWHM is the full-width at halfmaximum. Columns 5 and 6 give the mean, $\mu_{\Sigma_{\mathrm{B}}}$, and standard deviation, $\sigma_{\Sigma_{\mathrm{B}}}$, of $\log _{10}\left(\Sigma_{\mathrm{B}} / \mathrm{M}_{\odot} \mathrm{pc}^{-2}\right)$, where $\Sigma_{\mathrm{B}}$ is the background surface density. Columns 7 and 8 give the mean, $\mu_{\Sigma_{0}}$, and standard deviation, $\sigma_{\Sigma_{0}}$, of $\log _{10}\left(\Sigma_{\mathrm{O}} / \mathrm{M}_{\odot} \mathrm{pc}^{-2}\right)$, where $\Sigma_{\mathrm{O}}$ is the spinal surface density. Columns 9 through 11 refer to the moments of the distribution of profile parameters on the $\left(\sigma_{\Sigma_{\mathrm{B}}}, \sigma_{\Sigma_{\mathrm{O}}}\right)$ plane: Column 9 gives the slope of the principal axis, columns 10 and 11 give, respectively, the major and minor axes of the moment ellipse.

\begin{tabular}{lcccccccccc}
\hline FIELD & $\mathcal{N}$ & $\mu_{\text {FWHM }}$ & $\sigma_{\text {FWHM }}$ & $\mu_{\Sigma_{\mathrm{B}}}$ & $\sigma_{\Sigma_{\mathrm{B}}}$ & $\mu_{\Sigma_{\mathrm{O}}}$ & $\sigma_{\Sigma_{\mathrm{O}}}$ & $S$ & $a$ & $b$ \\
\hline [0] ALL & 599 & -0.96 & 0.19 & 1.77 & 0.28 & 1.64 & 0.29 & 0.198 & 0.770 & 0.295 \\
[1] IC5146 & 59 & -0.80 & 0.17 & 1.49 & 0.14 & 1.52 & 0.32 & 5.82 & 0.558 & 0.225 \\
[2] ORION B & 234 & -0.82 & 0.12 & 1.70 & 0.22 & 1.54 & 0.33 & 1.88 & 0.641 & 0.250 \\
[3] AQUILA & 71 & -1.05 & 0.15 & 2.21 & 0.14 & 2.11 & 0.27 & 3.27 & 0.488 & 0.203 \\
[4] MUSCA & 10 & -1.10 & 0.24 & 1.72 & 0.10 & 1.43 & 0.18 & 3.86 & 0.327 & 0.153 \\
[5] POLARIS & 20 & -1.15 & 0.13 & 1.26 & 0.06 & 1.06 & 0.17 & 16.7 & 0.293 & 0.108 \\
[6] PIPE & 38 & -1.10 & 0.17 & 1.66 & 0.12 & 1.43 & 0.24 & 3.49 & 0.519 & 0.269 \\
[7] TAURUS L1495 & 110 & -1.15 & 0.20 & 1.76 & 0.16 & 1.67 & 0.30 & 5.02 & 0.519 & 0.269 \\
[8] OPHIUCHUS & 57 & -1.15 & 0.13 & 2.03 & 0.19 & 1.89 & 0.32 & 2.28 & 0.600 & 0.257 \\
& & & & & & & & & & \\
\hline
\end{tabular}

This paper has been typeset from a $\mathrm{T}_{\mathrm{E}} \mathrm{X} / \mathrm{L} \mathrm{T}_{\mathrm{E}} \mathrm{X}$ file prepared by the author. 